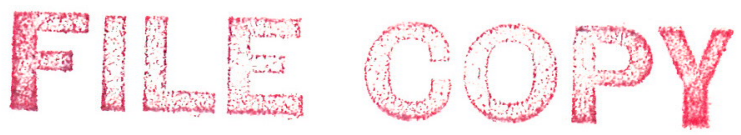

\author{
CERN - Data Handling Division \\ $\mathrm{DD} / 74 / 29$ \\ J.M.F. Chamayou \\ M.E.A. E1 Tom \\ 1 November 1974
}

ON THE APPROXTMATE SOLUTION OF THE DELAY INTEGRAL EQUATION

OF THE STATISTICAL THEORY OF RADIATION DAMAGE

(Submitted to Computer Physics Communications) 


\section{ON THE APPROXIMATE SOLUTION OF THE DELAY INTEGRAL EQUATION}

OF THE STATISTICAL THEORY OF RADIATION DAMAGE

J.M.F. Chamayou and M.E.A. E1 Tom

\section{ABSTRACT}

In the statistical theory of radiation damage, the mean number of atoms displaced in the atomic cascade is given by the delay integral equation

$$
\mu(x)=\int_{0}^{x} K(x, y)[\mu(y)+\mu(x-y-1)] d y, \quad x>0
$$

where $\mathrm{K}(\mathrm{x}, \mathrm{y})$ is a probability density function satisfying

$$
\begin{gathered}
\mathrm{K}(\mathrm{x}, \mathrm{y}) \geq 0, \quad \text { almost everywhere in } 0 \leq \mathrm{y} \leq \mathrm{x}<\infty \\
\int_{0}^{\mathrm{x}} \mathrm{k}(\mathrm{x}, \mathrm{y}) \mathrm{dy}=1,
\end{gathered}
$$

and where $\mu(x)$, the mean number of displaced atoms, satisfies the initial conditions

$$
\mu(x)= \begin{cases}0, & x<0 \\ 1, & 0 \leq x \leq 1 .\end{cases}
$$

Numerical procedures that use spline functions in conjunction with appropriate quadrature rules are presented for the construction of continuous approximations to $\mu(\mathrm{x})$. The methods presented are shown to be stable and to be of order $(m+1)$ for spline functions of degree $m$. Finally, the method for quadratic splines is used to compute $\mu(\mathrm{x})$ for atomic collisions with Firsov potentials, namely

$$
k(x, y)=\frac{1-s}{x}\left(\frac{x}{x-y}\right)^{s}, \quad 0 \leq y \leq x<\infty, \quad 0 \leq s<1,
$$

and with truncated Coulomb potentials, namely

$$
K(x, y)=\frac{\left(\beta^{2}-1\right)}{x[\beta-1+2(x-y) / x]^{2}}, \quad 0 \leq y \leq x<\infty, \quad 1<\beta<+\infty \text {. }
$$




\section{INTRODUCTION}

In this paper, we discuss approximate methods based on spline functions for the calculation of the average number of atoms displaced in a solid subject to neutron irradiation. This number, $\mu(x)$, is given by the following Volterra functional integral equation

$$
\mu(x)=\int_{0}^{x} K(x, y)[\mu(y)+\mu(x-y-1)] d y, \quad x>0
$$

where

$$
\mu(x)= \begin{cases}0, & x<0 \\ 1, & 0 \leq x \leq 1 ;\end{cases}
$$

$x$ and $y$ being, respectively, the energy of the primary atom before and after col1ision. Energy is expressed in units of displacement energy, namely

$$
\mathrm{x}=\mathrm{E} / \mathrm{E}_{\mathrm{d}},
$$

where $E_{d}$ is the energy lost by a secondary atom in freeing itself from the binding energy which holds it in the lattice, and $E$ is the energy of the primary atom.

The kernel $\mathrm{K}(\mathrm{x}, \mathrm{y})$ in $(1.1)$ is given by

$$
K(x, y)=\sigma(x, y) / \int_{0}^{x} \sigma(x, t) d t
$$

where $\sigma(x, y)$ is the differential cross-section of atomic collision. Thus $\mathrm{K}(\mathrm{x}, \mathrm{y})$ is a probability density function, namely

$$
\begin{aligned}
& \int_{0}^{x} K(x, y) d y=1, \\
& K(x, y) \geq 0, \quad 0 \leq y \leq x \\
& K(x, y) \neq 0, \quad \text { almost everywhere in } 0 \leq y \leq x<\infty .
\end{aligned}
$$

For the derivation of Eq. (1.1) and further details concerning the description of various models for the problem under consideration, we refer the reader to [1] and the references given there.

In Sections 3 and 4, we present numerical procedures for the computation of continuous approximate solutions for the second-kind Volterra equation 


$$
\mu(x)=g(x)+\int_{1}^{x} D(x, y) \mu(y) d y, \quad 1 \leq x \leq a
$$

where we assume

(A1) $g(x)$ is defined and integrable in the interval $[1, a]$,

(A2) $\mathrm{D}(\mathrm{x}, \mathrm{y})$ is bounded and integrable in the triangle $0 \leq \mathrm{y} \leq \mathrm{x} \leq \mathrm{a}$.

It is known (see, for example [2]) that under conditions (A1) and (A2), Eq. (1.2) has a unique bounded solution in $[1, a]$.

Observe that $\mathrm{Eq} .(1.1)$ is a special case of (1.2). Indeed, on taking

$$
D(x, y)= \begin{cases}K(x, y), & 1 \leq x \leq 2 \\ K(x, y)+H(x-y-1) K(x, x-y-1), & 2<x \leq a\end{cases}
$$

where $H(\cdot)$ is the Heaviside unit function, we obtain (1.1).

We write

$$
D^{*}=\max _{0 \leq y \leq x \leq a}|K(x, y)| ;
$$

introduce the mesh

$$
1=x_{1}<x_{2}<\ldots<x_{n}=a
$$

and denote by $h_{i}$ and $h$ the quantities

$$
\begin{aligned}
& \mathrm{h}_{i}=\mathrm{x}_{\mathrm{i}+1}-\mathrm{x}_{\mathrm{i}}, \quad \mathrm{i}=1(1) \mathrm{n}-1 \\
& \mathrm{~h}=\max _{\mathrm{i}} .
\end{aligned}
$$

In Section 2 below we discuss briefly the (cubic spline) method presented in [1]. In Sections 3 and 4, respectively, we present linear and quadratic spline function approximation methods and establish their convergence and stability properties. It is assumed there, however, that the kernel is bounded. In Section 5, it is shown how to modify the methods of Sections 3 and 4 in order to deal with the important case of unbounded kernels. Comments on the construction of methods based on higher order spline functions are given in Section 6 . Finally, in Section 7 , we consider some examples.

\section{RESUME OF THE APPROXIMATION METHOD USED IN [1]}

Although cubic spline interpolation is used in [1], here we shall, for simplicity, briefly outline the method for linear spline interpolation. This, it will be observed, is sufficiently adequate for our purpose of bringing out the main limitations of this method. 
Interpolate $\mu(x)$ at the mesh points (1.4) by the linear spline function $(\operatorname{see}[3])$

$$
\begin{gathered}
s(x)=\sum_{i=1}^{n} \mu_{i} C_{i}(x) \\
\mu_{i}=\mu\left(x_{i}\right), \quad i=1(1) n, \\
C_{i}\left(x_{j}\right)=\delta_{i, j}, \quad i, j=1(1) n
\end{gathered}
$$

where $\delta_{i j}$ is the Kronecker symbol. Define, for $i, j=1(1) n$, the quantities

$$
\left.\begin{array}{l}
I_{j, i}=\int_{1}^{x_{j}} K\left(x_{j}, y\right) C_{i}(y) d y \\
J_{j, i}=\left\{\begin{array}{l}
0, \\
\int_{j}^{0} x_{j} \leq 2
\end{array}\right. \\
f_{j}=\int_{0}^{1} k\left(x_{j}, x_{j}-y-1\right) C_{i}(y) d y, \quad x_{j}>2
\end{array}\right\}
$$

Then, in order to compute the $\mu_{i}$ in $(2.1)$, we require

$$
\mu_{j}=f_{j}+\sum_{i=0}^{n}\left(I_{j, i}+J_{j, i}\right) \mu_{i}, \quad j=1(1) n
$$

which gives rise, after some simple manipulations and in a self-explanatory notation, to the following linear system

$$
A \underline{\mu}=\underline{\hat{E}},
$$

where

$$
a_{i j}= \begin{cases}1-I_{i, j}, & i=j \\ -\left(I_{j, i}+J_{j, i}\right), & i \neq j\end{cases}
$$


Concerning the existence of an inverse of $A$, we have the following negative result. LEMMA I

The matrix $A$ has a strictly dominant diagonal only if $x \in[1,3]$. PROOF

We want to prove that

$$
\left|a_{j, j}\right|>\sum_{\substack{i=1 \\ i \neq \neq}}^{n}\left|a_{i, j}\right|, \quad j=1(1) n
$$

but

and

$$
a_{j, j}=1-\int_{x_{j-1}}^{x} k\left(x_{j}, y\right) \frac{\left(y-x_{j-1}\right)}{h_{j-1}} d y
$$

$\sum_{i=1}^{n}\left|a_{i, j}\right|=\sum_{i=1}^{j-1}\left(I_{j, j}+J_{j, i}\right)$,

$i \neq j$

$$
\begin{aligned}
& =\int_{x_{j-1}}^{x_{j}} k\left(x_{j}, y\right) \frac{\left(x_{j}-y\right)}{h_{j-1}} d y+\int_{1}^{x_{j-1}^{j}} k\left(x_{j}, y\right) d y+\int_{1}^{x^{j-1}} k\left(x_{j}, x_{j}-y-1\right) d y \\
& j=2(1) n \text {. }
\end{aligned}
$$

Thus for (2.3) to hold we must have

$$
\int_{0}^{x_{j-1}} K\left(x_{j}, y\right) d y>\int_{2}^{x_{j}-1} K\left(x_{j}, x_{j}-y-1\right) d y+\int_{1}^{x_{j}-1} K\left(x_{j}, y\right) d y,
$$

i.e. the diagonal is strictly dominant only if:

$$
\int_{0}^{1} K\left(x_{j}, y\right) d y>\int_{0}^{x_{j}^{-2}} K\left(x_{j}, y\right) d y
$$

Inequality (2.7) holds only for $2 \leq x_{j}<3$ and a fortioni for $1 \leq x_{j}<2$ 
More weak criteria for non-singularity may be found in [4], for instance Nekrassov's condition. But there will always exist a limit on the value of the argument $\mathrm{x}$ beyond which such criteria can no longer be guaranteed to hold.

It is apparent from the above analysis that the method suffers from certain drawbacks, which limit its range of applicability. These are:

i) in view of Lemma 1 , we are unable to guarantee that $(2.2)$ will have a (unique) solution beyond $\mathrm{x}=3$;

ii) in order to improve accuracy, a small $\mathrm{h}$ is required thus giving rise to a large system of equations with a dense matrix;

iii) for cubic splines, the approximate solution $S(x) \in C^{2}$, whereas the actual solution $\mu(\mathrm{x}) \in \mathrm{C}^{1}$.

A further drawback that is not revealed by the above analysis but has been pointed out in $[1]$ is:

iv) the method behaves badly for unbounded kernels.

\section{LINEAR SPLINE FUNCTION APPROXIMATION}

In this section we describe a method for constructing a linear spline function approximation $\mathrm{S}(\mathrm{x})$ and discuss its convergence and stability properties.

\subsection{Construction of $\mathrm{S}(\mathrm{x})$}

For $x \in\left[x_{i}, x_{i+1}\right], i=1, \ldots, n-1$, define $S(x)$ by

$$
\begin{aligned}
& S(x)=S\left(x_{i}\right)+\left(x-x_{i}\right) \alpha_{i} \\
& S\left(x_{1}\right)=\mu\left(x_{1}\right),
\end{aligned}
$$

where $\alpha_{i}$ is determined according to the relation (see Eq. (1.2)

$$
\begin{gathered}
S\left(x_{i+1}\right)=g\left(x_{i+1}\right)+T_{i}\left[D\left(x_{i+1}, y\right) S(y)\right], \quad i=1(1) n-1, \\
T_{i}[f(x)]=\frac{h}{2}\left[f\left(x_{1}\right)+2 \sum_{t=2}^{i} f\left(x_{t}\right)+f\left(x_{i+1}\right)\right] .
\end{gathered}
$$

Henceforward, we shall for simplicity assume the mesh (1.4) to be uniform, i.e. $h_{i}=h$ for all $i$. 


\section{THEOREM 1}

The construction (3.1)-(3.2) is well-defined and gives rise to a unique linear spline function $S(x)$, i.e. $S(x) \in C\left[x_{1}, x_{n}\right]$, for all $0<h<2 / D^{*}$.

\section{$\underline{P R O O F}$}

Equations (3.1) and (3.2) show that the coefficient of $\alpha_{i}$ is $1-\mathrm{hD}\left(\mathrm{x}_{\mathrm{i}+1}, \mathrm{x}_{\mathrm{i}+1}\right) / 2$.

Q.F.D.

\subsection{Order of convergence}

Here, in addition to assumptions (A1) and (A2) of Section 1, we shall require

(A3) $\mathrm{D}(\mathrm{x}, \mathrm{y})$ together with first- and second-order partial derivatives with respect to $y$ to be continuous in

$$
\mathrm{z}_{\mathrm{x}}=\left\{(\mathrm{x}, \mathrm{y}): \mathrm{x}_{1} \leq \mathrm{y}<\mathrm{x}-1\right\} \mathrm{U}\{(\mathrm{x}, \mathrm{y}): \mathrm{x}-1<\mathrm{y} \leq \mathrm{x}\}
$$

for each $x$ in $\left[x_{1}, a\right]$;

(A4) $\mu(x) \in C^{\prime}\left[x_{1}, a\right]$ and has a second derivative that is bounded in $\left[x_{1}, a\right]$ and continuous in $\Gamma=\left[x_{1}, a\right] \backslash\left\{y_{1}, y_{2}, \ldots, y_{N}\right\}, y_{i} \in\left[x_{1}, a\right], i=1(1) N$.

Observe that both assumptions are satisfied for Eq. (1.1) with $N=1, y_{1}=2$. We write

$$
e(x)=s(x)-\mu(x) \cdot
$$

On subtracting from (3.2) the result of applying, in a self-evident manner, the trapezoidal rule to Eq. (1.2), we obtain

$$
e\left(x_{i+1}\right)=T_{i}\left[D\left(x_{i+1}, y\right) e(y)\right]+R_{i},
$$

where $R_{i}=O\left(h^{2}\right)$ and depends only on $\mu(x)$ and $D(x, y)$.

Remark. In what follows we shall define

$$
\mu^{\prime \prime}(x)=\frac{1}{2}\left[\mu^{\prime \prime}(x+0)+\mu^{\prime \prime}(x-0)\right], \quad x=y_{i}, \quad i=1(1) N
$$

If we now set in (3.1)

$$
\begin{gathered}
\alpha_{i}=\mu^{\prime}\left(x_{i}\right)+h \beta_{i}, \\
\mu^{\prime}\left(x_{i}\right)=\mu^{\prime}\left(x_{1}+0\right), \quad i=1,
\end{gathered}
$$

where $\beta_{i}=\beta_{i}(h)$, we obtain on using Taylor's expansion for $\mu(x)$

$$
e(x)=e\left(x_{i}\right)+\left(x-x_{i}\right)\left\{h \beta_{i}-\frac{1}{2}\left(x-x_{i}\right) \mu^{\prime \prime}\left[\xi_{i}(x)\right]\right\}, \quad x_{i}<\xi_{i}(x)<x \leq x_{i+1} ;
$$


whence on differencing (3.4) for $i$ and $i+1$, we get

$$
\begin{aligned}
& h^{2}\left[1-h D\left(x_{i+1}, x_{i+1}\right) / 2\right]\left\{\beta_{i}-\frac{1}{2} \mu^{\prime \prime}\left[\xi_{i}\left(x_{i+1}\right)\right]\right\}= \\
& \quad=T_{i}^{*}\left[D\left(x_{i+1}, y\right) e(y)\right]-T_{i-1}\left[D\left(x_{i}, y\right) e(y)\right]+R_{i}-R_{i-1},
\end{aligned}
$$

where

$$
T_{i}^{*}[f(x)]=T_{i}[f(x)]-\frac{h}{2} f\left(x_{i+1}\right) .
$$

If we further set

$$
\beta_{i}=\frac{1}{2} \mu^{\prime \prime}\left[\xi_{i}\left(x_{i+1}\right)\right]+\beta_{i}^{*},
$$

then (3.6) gives

$$
\begin{aligned}
& e\left(x_{i+1}\right)=e\left(x_{i}\right)+h^{2} \beta_{i}^{*} \\
& e\left(x_{i}\right)=h^{2} \sum_{k=1}^{i-1} \beta_{k}^{*},
\end{aligned}
$$

since $e\left(x_{1}\right)=0$.

LEMMA 2

Let conditions (A1)-(A4) hold. Then, for a1l $i$, we have

$$
\beta_{i}^{*}=O(h) \text {, }
$$

$\underline{P R O O F}$

The proof is by induction. However, we first observe that $R_{1}$ as well as $R_{i}-R_{i-1}, i>1$, are $O\left(h^{3}\right)$ (see, for example, Krylov [5]).

For $i=1, e\left(x_{1}\right)=0$ and the result follows from (3.4). Now assume $(3.10)$ holds for $k=1, \ldots, i-1$. If we observe that the first two components of the right-hand side of (3.7) typically give terms of the form

$$
h\left[D\left(x_{i+1}, x_{t}\right)-D\left(x_{i}, x_{t}\right)\right] e\left(x_{t}\right), \quad t=2, \ldots, i-1,
$$

then it is not difficult to see, in view of the induction hypothesis and (3.9), that the right-hand side of (3.7) is $O\left(h^{3}\right)$. Thus (3.7) and (3.8) give $\beta_{i}^{*}=O(h)$.

Q.E.D.

As an immediate consequence of Lemma 2 and (3.6) we have 


\section{THEOREM 2}

Let conditions (A1)-(A4) be satisfied. Then the error (3.3) satisfies

$$
\begin{array}{ll}
e(x)=o\left(h^{2}\right), & x \in\left[x_{1}, x_{n}\right] \\
e^{\prime}(x)=o(h), & x \in\left[x_{1}, x_{n}\right] \\
e^{\prime \prime}(x)=o(1), & x_{1}<x<x_{n}
\end{array}
$$

where

$$
S^{(r)}\left(x_{i}\right)=\left[S^{(r)}\left(x_{i}+h / 2\right)+S^{(r)}\left(x_{i}-h / 2\right)\right] / 2, \quad r=1,2 .
$$

\subsection{Numerica1 stability}

Since $S(x)$ is constructed in a stepwise fashion, we have to ensure that errors do not build up. We shall say that the method is numerically stable if all solutions $\left\{\mathrm{S}\left(\mathrm{x}_{\mathrm{i}}\right)\right\}$ remain bounded as $\mathrm{i} \rightarrow \infty, \mathrm{h} \rightarrow 0\left(\mathrm{x}_{\mathrm{i}} \mathrm{fixed}\right)$ when it is applied to the integral equation

$$
\begin{aligned}
\mu(x)=g(x)+\int_{1}^{x} E(x-1-y) \mu(y) d y \\
E(z)= \begin{cases}p, & z \geq 0 \\
q, & z<0 .\end{cases}
\end{aligned}
$$

Application of (3.2) to (3.12) yields (for all $\mathrm{h}<1$ )

$$
S\left(x_{i+1}\right)-S\left(x_{i}\right)=c_{i}+q h\left[S\left(x_{i}\right)+h \alpha_{i} / 2\right] \text {, }
$$

where

$$
c_{i}=g\left(x_{i+1}\right)-g\left(x_{i}\right)+\int_{x_{1}}^{x_{i}}\left[E\left(x_{i+1}-1-y\right)-E\left(x_{i}-1-y\right)\right] S(y) d y
$$

Since the left-hand side of (3.13) is simply h $\alpha_{i}$, we easily obtain from (3.1) and (3.13)

$$
S\left(x_{i+1}\right)=w S\left(y_{i}\right)+c_{i} /(1-q h / 2)
$$

where

$$
w=(1+q h / 2) /(1-q h / 2)
$$

from which immediately follows 


\section{THEOREM 3}

The linear spline function approximation method (3.1)-(3.2) is numerically stable.

Remark. In fact the method is A-stable in the sense defined in [6].

\section{QUADRATIC SPLINE FUNCTION APPROXIMATION}

We now describe a method for constructing a quadratic approximating function $S(x) \in C^{\prime}\left[x_{1}, x_{n}\right]$. Since the proofs of the existence and uniqueness, convergence and stability results are similar to those of the corresponding results of Section 3, we sha11 omit them.

\subsection{Construction of $S(x)$}

$$
\text { For } \begin{aligned}
x \in\left[x_{i}, x_{i+1}\right], & i=1, \ldots, n-1, \text { define } s(x) \text { by } \\
S(x) & =S\left(x_{i}\right)+\left(x-x_{i}\right) S^{\prime}\left(x_{i}\right)+\left(x-x_{i}\right)^{2} \alpha_{i}, \\
S\left(x_{1}\right) & =\mu\left(x_{1}\right) \\
S^{\prime}\left(x_{1}\right) & =\mu\left(x_{1}+0\right),
\end{aligned}
$$

where $\alpha_{i}$ is determined according to the relation

$$
\begin{aligned}
& S\left(x_{i+1}\right)=g\left(x_{i+1}\right)+Q_{i}\left[D\left(x_{i+1}, y\right) S(y)\right] \\
& Q_{i}[f(x)]=\frac{h}{6}\left[f\left(x_{1}\right)+4 \sum_{j=1}^{i} f\left(x_{j}+h / 2\right)+2 \sum_{j=2}^{i} f\left(x_{j}\right)+f\left(x_{i+1}\right)\right] .
\end{aligned}
$$

\section{THEOREM 4}

The construction (4.1)-(4.2) defines $S(x)$ uniquely as a quadratic spline function in $\left[\mathrm{x}_{1}, \mathrm{x}_{\mathrm{n}}\right]$ for all $0<\mathrm{h}<6 / \mathrm{D}^{*}$.

\subsection{Order of convergence}

We continue to denote by $z_{x}$ and $\Gamma$ the regions defined in Section 3.2 above. We further assume that

(A5) $D(x, y)$ together with its partial derivative through order four with respect to $y$ are continuous in $z_{x}$ for each $x \in\left[x_{1}, a\right]$;

(A6) $\mu(x) \in C^{4}(\Gamma)$ and has derivatives through order four that are bounded at $\mathrm{y}_{1}, \mathrm{y}_{2}, \ldots, \mathrm{y}_{\mathrm{N}}$. 
Remark 1. The motivation behind condition (A6) is our knowledge that the solution $\mu(x)$ of $E q .(1.1)$ is in $C^{m}(m, \infty), m=0(1) n+1$, provided that $K(x, y) \in C^{n}$ in $0<\mathrm{y} \leq \mathrm{x}<\infty$.

Remark 2. As in Section 3, we define $\mu^{(r)}(x)=\left[\mu^{(r)}(x+0)+\mu^{(r)}(x-0)\right] / 2, \quad x=y_{i}, \quad i=1(1) N ; \quad r=2,3,4$.

For the proof of the result corresponding to Theorem 2 above, it is necessary to deal with the intervals $\left[x_{1}, x_{i}-x_{1}\right],\left[x_{i}-x_{1}, x_{i}\right]$ as well as $\left[x_{1}, 2\right],[2,3]$, $[3,4],\left[4, x_{i}\right]$ (assuming $x_{i}>4$ ) separately. This is so since the expression for the remainder in Simpson's rule requires the existence of the fourth derivative of the integrand. Bearing this in mind and arguing as in Section 3.2 above, we obtain

\section{THEOREM 5}

Let conditions (A1), (A2), (A4)-(A6) be satisfied. Then the error(3.3), where $S(x)$ is defined by $(4.1)-(4.2)$, satisfies

$$
\begin{aligned}
e(x) & =o\left(h^{3}\right), & & x \in\left[x_{1}, x_{n}\right] \\
e^{\prime}(x) & =0\left(h^{2}\right), & & x \in\left[x_{1}, x_{n}\right] \\
e^{\prime \prime}(x) & =o(h), & & x_{1}<x<x_{n}
\end{aligned}
$$

where $S^{\prime \prime}(x)$ is as defined in Theorem 2 above.

\subsection{Numerical stability}

Define the column vector

$$
\underline{S}_{t}=\left[\begin{array}{ll}
S\left(x_{t}\right) & h S^{\prime}\left(x_{t}\right)
\end{array}\right]^{T} .
$$

Then (4.1) gives

$$
\underline{S}_{i+1}=\left(\begin{array}{ll}
1 & 1 \\
0 & 1
\end{array}\right) \underline{S}_{i}+\left(\begin{array}{l}
1 \\
2
\end{array}\right) h^{2} \alpha_{i} .
$$

If we now apply (4.2) to (3.12) in the manner of Subsection 3.3 above, we find in view of (4.3) and after some simple manipulations

$$
\underline{s}_{i+1}=\underline{W S}_{i}+\left(\begin{array}{l}
1 \\
2
\end{array}\right) c_{i} /(1-v)
$$

where $c_{i}$ is given by (3.14) and where 


$$
\begin{aligned}
& -11- \\
& \mathrm{W}=\frac{1}{1-\mathrm{v}}\left(\begin{array}{cc}
1+2 \mathrm{v} & \mathrm{v} / 2 \\
6 \mathrm{v} & 2 \mathrm{v}-1
\end{array}\right) \\
& \mathrm{v}=\mathrm{qh} / 3,
\end{aligned}
$$

and inasmuch as the eigenvalues $\lambda$ of $w$ are given by

$$
\left.\lambda=\left[2 \mathrm{v} \pm \sqrt{(1}+3 \mathrm{v}^{2}\right)\right] /(1-\mathrm{v})
$$

we have

\section{THEOREM 6}

The quadratic spline function approximation method (4.1)-(4.2) is numerically stable.

\section{CASE OF UNBOUNDED KERNEL}

Of significance, in the statistical theory of damage, is the case of Firsov potentials

$$
D(x, y)=(x-y)^{-s_{D}}(x, y), \quad 0 \leq s<1
$$

where $D_{s}(x, y)$ satisfies condition (A1) of Section 1 . Evidently, the methods of Section 3 and 4 are no longer applicable in this case. However, we shall briefly outline in this section applicable modifications of these methods so that, in particular, the convergence and stability results of previous sections remain valid for the modified method. It suffices to outline the modification for the linear case.

Let $S(x)$ be defined by (3.1) where $\alpha_{i}$ is so determined that

$$
\begin{aligned}
& S\left(x_{i+1}\right)=g\left(x_{i+1}\right)+L_{i}\left[D\left(x_{i+1}, y\right) S(y)\right], \quad i=1(1) n-1 \\
& L_{i}[f(x)]=\sum_{j=1}^{i} \int_{x_{j}}^{x_{j+1}} f(x) d x .
\end{aligned}
$$

Remark. Instead of performing exact integration (assuming it to be possible) we may choose to apply an appropriate quadrature rule in the interval $\left[x_{1}, x_{i+1}-1\right]$ together with a Gaussian rule

$$
\int_{0}^{1} x^{-S} f(x) d x \approx \sum_{i=1}^{m} w_{i} f\left(x_{i}\right)
$$


in the interval $\left[x_{i+1}-1, x_{i+1}\right]$. Weights and nodes for the rules (5.2) are tabulated in $[7]$ for $S=-3.0(0.1) 0.9, \mathrm{~m}=1(1) 8$, to eight significant figures.

Remark. For the quadratic case $S(x)$ is defined by (4.1) and, in general, we do not know $\mu^{\prime}\left(x_{1}+0\right)$. An approximate value, however, may be obtained by applying initially the linear spline function method of section 3 in the following way. Set

$$
\begin{aligned}
& \alpha_{1}:=0 \\
& \mathrm{x}_{2}:=\mathrm{h} / 2, \\
& \mathrm{x}_{2}:=\mathrm{x}_{2} / 2 ;
\end{aligned}
$$

apply the method of Section 3 in $\left[\mathrm{x}_{1}, \mathrm{x}_{2}\right]$ to obtain $\alpha_{2}$. Repeat until $\left|\alpha_{1}-\alpha_{2}\right|<\varepsilon$ for some prescribed $\varepsilon$.

\section{HIGHER ORDER SPLINE FUNCTION APPROXIMATIONS}

In order to achieve higher accuracy, one may want to construct, in the manner of Sections 3-5, methods for splines of degree $m \geq 3$. However, it has recently been shown in [6] that such methods are numerically unstable for all m $\geq 3$. Notwithstanding this negative result, it is also shown in [6] that stable methods may be constructed for spline functions of degree $m$ deficiency $(m-1)$, i.e. in the continuity class $\mathrm{c}^{\mathrm{m}-2}$. Such methods, however, require the solution of $(\mathrm{m}-1)$ linear equations per step. For a detailed description of the method for the case $\mathrm{m}=3$, we refer the reader to $[8]$. This latter method is fourth order.

\section{EXAMPLES}

Three examples are considered. We used the first in order to test the accuracy of the method and the second and the third to compare our results with those of Robinson [9] for the kernels

$$
\begin{gathered}
\mathrm{K}(\mathrm{x}, \mathrm{y})=\frac{1-\mathrm{s}}{\mathrm{x}}\left(\frac{\mathrm{x}}{\mathrm{x}-\mathrm{y}}\right)^{\mathrm{s}}, \quad 0 \leq \mathrm{y} \leq \mathrm{x}<\infty, \quad 0 \leq \mathrm{s}<1 \\
\mathrm{~K}(\mathrm{x}, \mathrm{y})=\left(\beta^{2}-1\right) /\left\{\mathrm{x}[\beta-1+2(\mathrm{x}-\mathrm{y}) / \mathrm{x}]^{2}\right\}, \quad 0 \leq \mathrm{y} \leq \mathrm{y}<\infty, \quad 1<\beta<+\infty .
\end{gathered}
$$

The test example is for the classical hard spheres potential, namely

$$
\mathrm{K}(\mathrm{x}, \mathrm{y})=\frac{1}{\mathrm{x}}, \quad 0 \leq \mathrm{y} \leq \mathrm{x}<\infty, \quad \text { i.e. } \mathrm{s}=0 \text { and } \beta \rightarrow+\infty \text {. }
$$

In Table 1 we give the exact and calculated values for $\mu(x)$, and we compare our results with those of Lal $[10]$. 
Robinson [9] has given an analytical expression for the asymptotic solution of the following equation without delay of the radiation damage for the kernels $(7.1)$ and $(7.2)$

$$
v(x)=\int_{0}^{x} k(x, y)[v(y)+v(x-y)] d y
$$

where

$$
\begin{array}{ll}
v(x)=0, & 0 \leq x \leq 1 \\
v(x)=1, & 1 \leq x \leq 2,
\end{array}
$$

$v(x)$ being the mean number of atoms displaced by a primary of energy $x$. Note that his model is different from that considered in the present paper, namely the one described by Eq. (1.1).

Figure 1 shows that when $s$ tends to 1 the two models tend to coincide. Indeed, we have the following relations.

$$
\begin{aligned}
\text { For } s=0, \quad \lim _{x \rightarrow \infty} \mu^{\prime}(x) & =e^{-\gamma}=0.561, \quad \gamma=\text { Euler's constant } \\
\nu^{\prime}(x) & =0.5 \\
\text { For } s \rightarrow 1, \quad \lim _{x \rightarrow \infty} \mu^{\prime}(x) & =0.43 \\
\lim _{x \rightarrow \infty} \nu^{\prime}(x) & =\frac{61 n 2}{\pi^{2}}=0.42,
\end{aligned}
$$

the extremal value $s \rightarrow 1$, has the following meaning. The primary suffers a number of shocks that tend to infinity without exchange of energy and it loses its energy in a finite number of shocks, some of which may generate secondary atoms.

Figure 2 represents the variation of the displacement coefficient

$$
A(\beta)=\frac{\mu(x)}{1+x}
$$

for the truncated Coulomb potential (7.2). $\quad \beta \rightarrow 1$ gives the Rutherford crosssection. It can be seen in [9] that the parameter $\beta$ can be related to Bohr and Born-Mayer potentials. 
$\underline{\text { Table } 1}$

Computed results for hard sphere case $(h=0.02)$

\begin{tabular}{|c|c|c|c|c|}
\hline $\mathrm{x}$ & $\begin{array}{l}\mu(\mathrm{x}) \\
\operatorname{exact}\end{array}$ & $\begin{array}{c}\mu(x) \\
\text { calculated }\end{array}$ & $\begin{array}{c}\mu^{\prime}(\mathrm{x}) \\
\text { Lal's results }\end{array}$ & $\begin{array}{c}\mu^{\prime}(x) \\
\text { calculated }\end{array}$ \\
\hline 1 & 1 & 1 & 1 & 0.990082 \\
\hline 1.1 & 1.0953102 & 1.0953092 & & \\
\hline 1.2 & 1.1823215 & 1.1823210 & & \\
\hline 1.3 & 1.2623642 & 1.2623632 & & \\
\hline 1.4 & 1.3364722 & 1.3364716 & $\underline{1}$ & \\
\hline 1.5 & 1.4054651 & 1.4054640 & $x$ & \\
\hline 1.6 & 1.4700036 & 1.4700028 & & \\
\hline 1.7 & 1.5306282 & 1.5306270 & & \\
\hline 1.8 & 1.5877866 & 1.5877857 & & \\
\hline 1.9 & 1.6418539 & 1.6418526 & & \\
\hline 2.0 & 1.6931472 & 1.6931461 & 0.500000 & 0.502529 \\
\hline 2.1 & 1.7442804 & 1.7442788 & & \\
\hline 2.2 & 1.7972718 & 1.7972706 & 0.537418 & 0.536069 \\
\hline 2.3 & 1.8516176 & 1.8516159 & & \\
\hline 2.4 & 1.9069287 & 1.9069273 & 0.556863 & 0.556210 \\
\hline 2.5 & 1.9629010 & 1.9628991 & & \\
\hline 2.6 & 2.0192953 & 2.0192937 & 0.565386 & 0.565164 \\
\hline 2.7 & 2.0759221 & 2.0759200 & & \\
\hline 2.8 & 2.1326303 & 2.1326286 & 0.567067 & 0.567117 \\
\hline 2.9 & 2.1892995 & 2.1892974 & & \\
\hline 3 & 2.2458324 & 2.2458310 & 0.564382 & 0.564607 \\
\hline 3.1 & 2.3021774 & 2.3021764 & & \\
\hline 3.2 & 2.3583885 & 2.3583878 & 0.561647 & 0.561739 \\
\hline 3.3 & 2.4145224 & 2.4145215 & & \\
\hline 3.4 & 2.4706182 & 2.4706174 & 0.560861 & 0.560885 \\
\hline 3.5 & 2.5267015 & 2.5267003 & & \\
\hline 3.6 & 2.5827879 & 2.5827869 & 0.560915 & 0.560913 \\
\hline 3.7 & 2.6388865 & 2.6388851 & & \\
\hline 3.8 & 2.6950005 & 2.6949992 & 0.561218 & 0.561212 \\
\hline 3.9 & 2.7511296 & 2.7511280 & & \\
\hline 4.0 & 2.8072708 & 2.8072694 & 0.561458 & 0.561458 \\
\hline
\end{tabular}


$\underline{\text { Table } 2}$

Variance $\sigma^{2}(x)$ for hard spheres $(h=0.02)$

\begin{tabular}{|c|c|c|}
\hline $\mathrm{x}$ & $\begin{array}{c}\sigma^{2}(\mathrm{x}) \\
\text { exact }\end{array}$ & $\begin{array}{c}\sigma^{2}(\mathrm{x}) \\
\text { calculated }\end{array}$ \\
\hline 3.0 & 0.28262 & 0.28261 \\
3.1 & 0.28982 & 0.28981 \\
3.2 & 0.29566 & 0.29567 \\
3.3 & 0.30091 & 0.30087 \\
3.4 & 0.30606 & 0.30603 \\
3.5 & 0.31143 & 0.31140 \\
3.6 & 0.31718 & 0.31715 \\
3.7 & 0.32335 & 0.32334 \\
3.8 & 0.32995 & 0.32995 \\
3.9 & 0.33688 & 0.33687 \\
4.0 & 0.34404 & 0.34403 \\
\hline
\end{tabular}

The variance $\sigma^{2}(x)$ of the number of displaced atoms is given by the following integral equation, which is of the same type as (1.1)

$$
\begin{aligned}
\sigma^{2}(x)= & \int_{0}^{x} K(x, y)\left[\sigma^{2}(y)+\sigma^{2}(x-y-1)\right] d y+\int_{0}^{x} K(x, y)[\mu(x)- \\
& -\mu(x-y-1)-\mu(y)]^{2} d y, \quad x \geq 1
\end{aligned}
$$

where

$$
\sigma^{2}(x)=0, \quad x \leq 1
$$

Figure 3 gives the variations of $\sigma^{2}(x)$ for the Firsov potential.

Figure 4 gives the variance for the truncated Coulomb potential.

We may observe as noted in $[1]$

$$
\lim _{x \rightarrow \infty}\left[\sigma^{2}(x)\right]^{\prime}=\text { constant }=\sigma_{a}^{2 \prime}
$$

For instance for the hard spheres potential

$$
\sigma_{a}^{2 \prime}=e^{-\gamma}\left(2 e^{-\gamma}-1\right)=0.069014 \text {, }
$$

the calculated result is 0.0685 . 
Table 2 presents for hard spheres the exact and the calculated variance for $x \in[3,4]$.

We can observe that as the anisotropy of the cross-section increases $(\mathrm{s} \rightarrow 1, \beta \rightarrow 1)$ the variance increases as pointed out in [1].

\section{CONCLUSION}

A numerical method has been presented to solve the equation of the statistical theory of damage; this method can be applied even if the cross-sections of atomic collisions are unbounded. The quadratic splines functions used have the required properties of convergence and stability. Test examples exhibit the accuracy of the method and its convenience for meaningful physical cases. 


\section{REFERENCES}

[1] J.M.F. Chamayou, J. Comput. Phys. 13, 70 (1973).

[2] W. Pogorzelski, Integral equations and their applications (Pergamon Press, New York, 1966), Vo1. 1.

[3] J.H. Ahlberg, E.N. Nilson and J.L. Walsh, The theory of splines and their applications (Academic Press, New York, 1967).

[4] D.W. Bailey and D.E. Crabtree, Linear Algebra Applic. 2, 303 (1969).

[5] V.I. Krylov, Approximate calculation of integrals (Macmillan, New York, 1962)

[6] M.E.A. E1 Tom, BIT 14, 136 (1974).

[7] V.I. Krylov et al., Tables for numerical integration of functions with power singularities

$$
\int_{0}^{1} x^{\beta}(1-x)^{\alpha} f(x) d x
$$

(Izdat. Akad. Nauk BSSR, Minsk, 1963).

[8] M.E.A. E1 Tom, BIT 13, 1 (1973).

[9] M.T. Robinson, Philos. Mag. 12, 741 (1965).

[10] M. Lal and P. Gillard, Numerical solution of two differential-difference equations of analytic theory of numbers, Conf. on the Numerical Solution of Differential Equations, Dundee, Scotland, June 1969, Lecture Notes in Mathematics No. 109 (Springer Verlag, Berlin, 1969). 


\section{Figure captions}

Fig. 1 : A(s): displacement coefficient for Firsov's potentials.

Fig. 2 : $\mathrm{A}(\beta)$ : displacement coefficient for the truncated coulomb potential.

Fig. 3 : Variance for the Firsov potential.

Fig. 4 : Variance for the truncated Coulomb potential. 




Fig. 1 


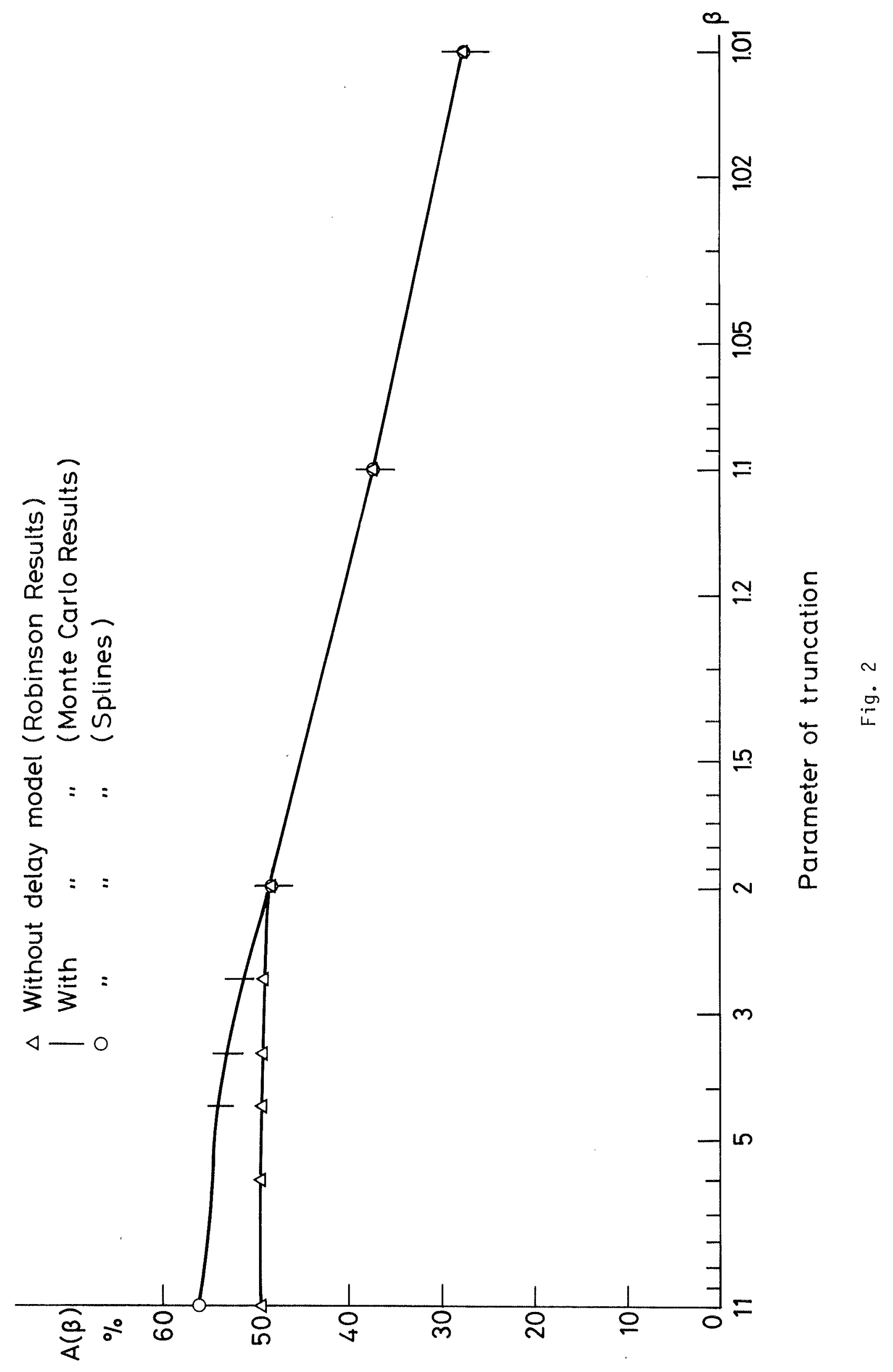




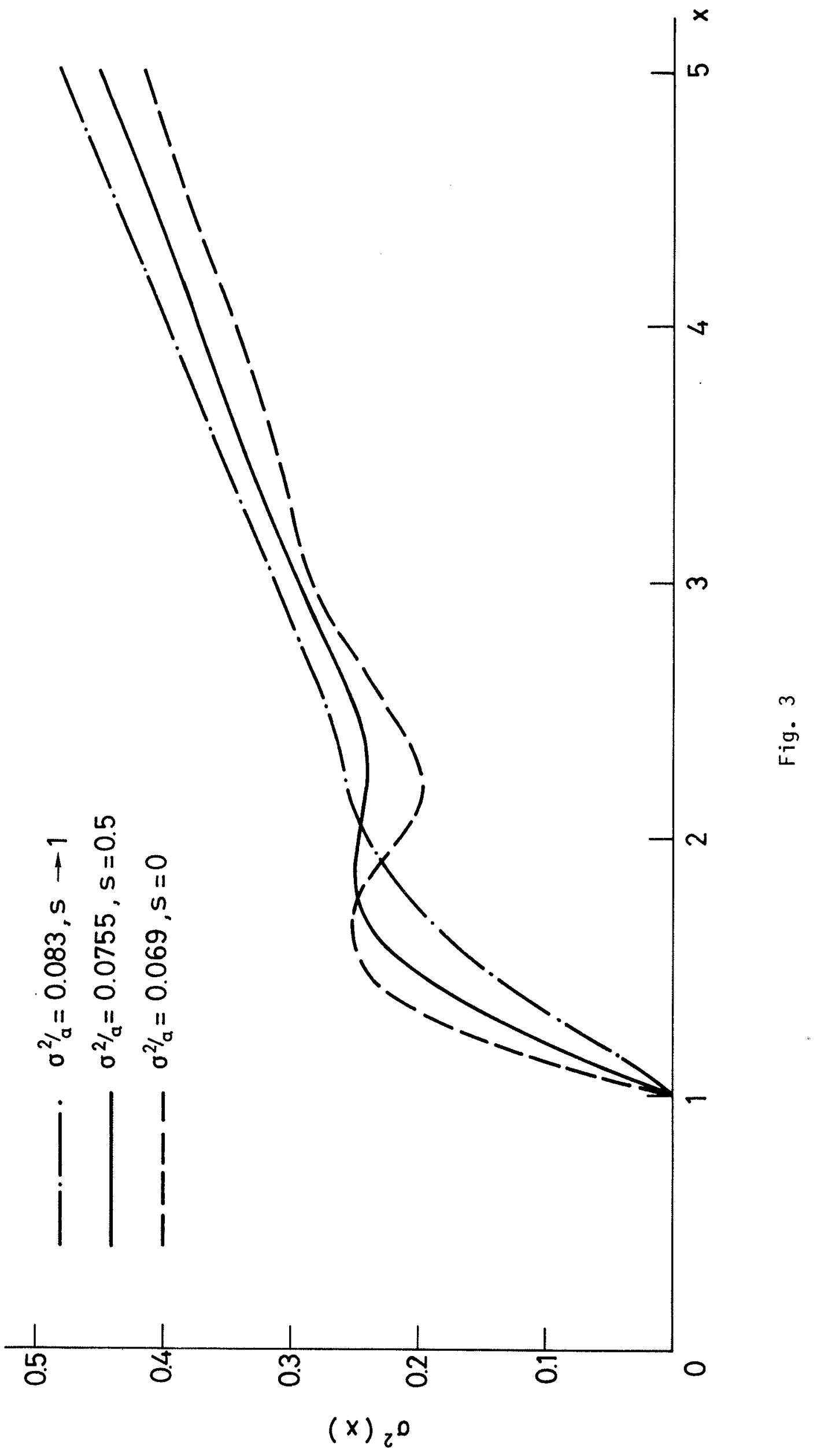




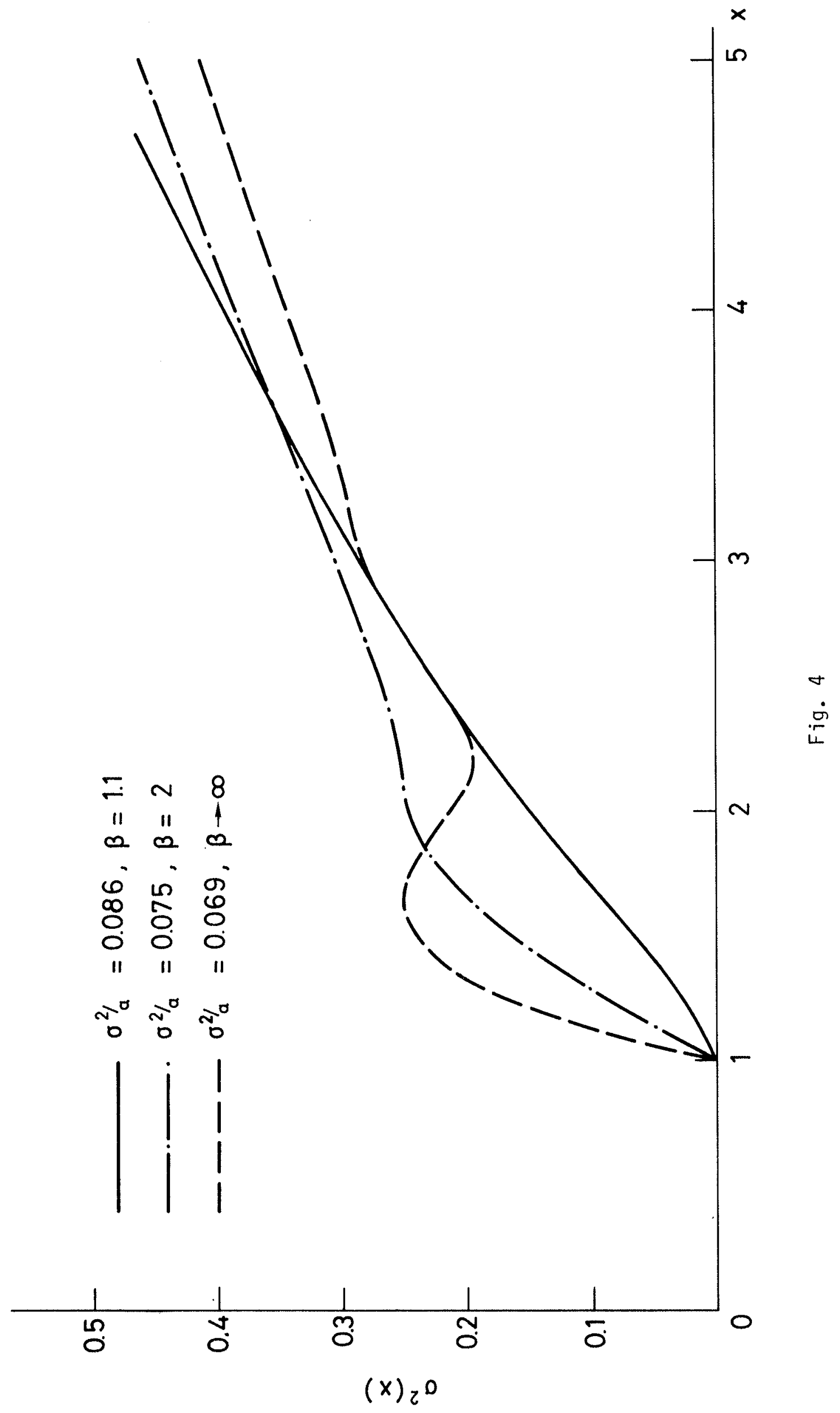

